\title{
Unreliability of cardiothoracic ratio as a marker of left ventricular impairment: comparison with radionuclide ventriculography and echocardiography
}

\author{
Andrew L Clark, Andrew J S Coats
}

\begin{abstract}
Background-The size of the heart assessed by cardiothoracic ratio on chest radiography is often used as a screening test for the presence of heart failure and for assessing its severity.

Methods-We compared cardiothoracic ratio (CTR), left ventricular ejection fraction (LVEF) from radionuclide ventriculography, and left ventricular dimensions from echocardiography in a population of 91 patients (aged 60.4 (SD 9.6) years) with a diagnosis of chronic heart failure.

Results-There was a weak relation between CTR and LVEF $(R=0.33)$ and fractional shortening from echocardiography $(R=0.22)$. LVEF and fractional shortening correlated more closely $(R=0.55)$. No measure of left ventricular function correlated with exercise capacity as measured by peak oxygen consumption. For the group of patients with a normal fractional shortening $(n=17)$, the left ventricle was dilated in all but two (mean end diastolic dimension $5.9(0.7) \mathrm{cm})$. The two with normal dimensions had a low ejection fraction. For the 12 patients with a CTR in the normal range, the left ventricular end diastolic dimension was only slightly smaller than for the rest $(6.2(0.9) v 6.9$ (1.2); $p=0.045)$.
\end{abstract}

Conclusions-Chest radiography is not a reliable indicator of the degree of left ventricular dysfunction. Echocardiography and radionuclide ventriculography are more appropriate investigations for assessing cardiac function. (Postgrad Med f 2000;76:289-291)

Academic Unit, Department of Cardiology, Castle Hill Hospital, Castle Road, Cottingham, Kingston-upon-Hull HU16 5JQ, UK A L Clark

Department of Cardiac Medicine, Imperial College School of Medicine, London, UK

A J S Coats

Correspondence to:

Dr Clark

(e-mail: A.L.Clark@

medschool.hull.ac.uk)

Submitted 16 September 1999

Accepted 27 October 1999
Although heart failure is common, the diagnosis can be difficult. Scoring systems have been described (primarily for use in epidemiological studies), ${ }^{13-5}$ which use the cardiothoracic ratio from a posteroanterior chest radiograph as an index of the size of the heart. The chest radiograph is widely available and frequently performed as a screening test for left ventricular failure. Other methods for assessing cardiac function, such as left ventricular angiography and echocardiography are less widely available.

Even after the diagnosis of heart failure is made, quantification of the severity of left ventricular impairment is important to give prognostic information. ${ }^{6} 7$

We sought to compare methods of quantifying left ventricular function in widespread use that are easily available in district general hospitals. We thus compared LVEF derived from radionuclide ventriculography with echocardiographic indices derived from $M$ mode echocardiography in patients with clinical heart failure. In particular, we were concerned to see whether the chest radiograph could be recommended as a method for assessment of left ventricular function.

\section{Methods}

This was a retrospective study. Ninety one patients from the Royal Brompton heart failure database were identified who had had a chest radiograph, radionuclide ventriculography, and an echocardiographic study within three months of each other. Only those patients were included for whom $M$ mode echocardiographic tracings were available.

\section{RADIONUCLIDE STUDY}

Subjects were injected intravenously with 0.03 $\mathrm{mg} / \mathrm{kg}$ of stannous fluoride red cell labelling agent (Amersham International). After allowing a period of 15-30 minutes for equilibration of the red blood cells, the patient was positioned supine on a scanning couch, with the gamma camera, a Sopha medical DS-X rectangular field of view, connected to lead V6 of an electrocardiograph to synchronise events. A bolus of $740 \mathrm{MBq}$ of ${ }^{99 m}$ technetium in $0.3-0.5 \mathrm{ml}$ was injected intravenously, with the gamma camera positioned at $45^{\circ}$ left anterior oblique with $10^{\circ}$ of craniocaudal tilt. An equilibrium radionuclide ventriculogram was then acquired; 16 frames were acquired in each R-R interval, and summed repeatedly, until the end diastolic image contained 300000 counts, to ensure an accurate estimation of LVEF. Ventricular ejection fraction was calculated by comparing background subtracted images at end diastole and end systole as: ejection 
Table 1 Demographic data $(n=91)$; results are number or mean (SD)

\begin{tabular}{ll}
\hline Age (years) & $60.4(9.6)$ \\
NYHA & 16 \\
I & 34 \\
II & 28 \\
III & 13 \\
IV & $86: 5$ \\
Male:female & $1.73(0.1)$ \\
Height $(\mathrm{m})$ & $79.9(14.5)$ \\
Weight $(\mathrm{kg})$ & 51 \\
Diagnosis & 40 \\
IHD & $18.2(7.1)$ \\
DCM & \\
Peak oxygen consumption $(\mathrm{ml} / \mathrm{min} / \mathrm{kg})$ & \\
\hline
\end{tabular}

DCM = dilated cardiomyopathy; $\mathrm{IHD}=$ ischaemic heart disease; NYHA = New York Heart Association classification of symptoms.

Table 2 Indices of left ventricular function; results are mean (SD)

\begin{tabular}{lll}
\hline Echo & LVEDD $(\mathrm{cm})$ & $6.77(1.17)$ \\
& LVESD $(\mathrm{cm})$ & $5.61(1.38)$ \\
& Fractional shortening & $0.18(0.09)$ \\
RNVG & LVEF $(\%)$ & $27.0(15.0)$ \\
CXR & CTR & $0.56(0.06)$ \\
\hline
\end{tabular}

$\mathrm{CTR}=$ cardiothoracic ratio $\mathrm{CXR}=$ chest radiograph $\mathrm{LVEDD}$ $=$ left ventricular end diastolic dimension; $\mathrm{LVEF}=$ left ventricular ejection fraction; LVESD = left ventricular end systolic dimension; RNVG = radionuclide ventriculography.

fraction $(\mathrm{LVEF})=($ end diastolic - end systolic counts)/end diastolic counts $\times 100$.

ECHOCARDIOGRAPHIC STUDY

Left ventricular internal dimensions were acquired from standard $M$ mode echocardiographic images in the parasternal long axis view at the mitral valve tips. Fractional shortening was calculated as left ventricular end diastolic dimension (LVEDD) minus left ventricular end systolic dimension (LVESD) divided by LVEDD.

CARDIOTHORACIC RATIO (CTR)

CTR was determined by one of us (ALC) only where a standard posteroanterior chest film from within three months of the radionuclide and echocardiographic studies was available. Only posteroanterior erect radiographs were accepted. No anteroposterior, supine, or seated films were accepted. The measurements were made blind to the results of the other investigations.

EXERCISE DATA

Exercise data were available from maximal incremental exercise tests with metabolic gas exchange data. Patients were encouraged to exercise to exhaustion using the standard Bruce protocol with the addition of a stage 0 (three minutes' exercise at one mile per hour with a 5\% gradient). Subjects breathed through a one way valve allowing the collection of expired air. Expired air was mixed with an inert indicator gas in a mixing chamber, and samples taken every 10 seconds to be analysed by mass spectrometer (Amis 2000, Odennse, Denmark). Peak oxygen consumption was derived.

STATISTICS

Results are shown as mean (SD). The relation between variables was explored using linear regression analysis.

\section{Results}

Demographic data are shown in table 1 . The average dose of frusemide (furosemide) being taken was 83 (55) mg daily, although seven were on no diuretic. Twenty one patients were not receiving angiotensin converting enzyme inhibitors. Twenty eight were receiving digoxin.

The indices of left ventricular function are shown in table 2. LVEDD and LVESD correlated closely $(R=0.95 ; \mathrm{p}<0.00001)$. The relations between LVEF from the radionuclide study and echocardiographic measurements and cardiothoracic ratio is shown in table 3 and figs 1 and 2 .

From fig 1, it can be seen that most patients have both abnormal LVEF and fractional shortening. For the group of patients with a normal fractional shortening $(n=17)$, the left ventricle was dilated in all but two (mean end diastolic dimension $5.9(0.7) \mathrm{cm})$. The two with normal dimensions had a low ejection fraction.

Table 3 Relations between the different measures of left ventricular function

\begin{tabular}{lllll}
\hline LVEDD & LVESD & Fractional shortening & LVEF \\
LVEF & $R=-0.48$ & $R=-0.56$ & $R=0.55$ & - \\
& $\mathrm{p}<0.00001$ & $\mathrm{p}<0.00001$ & $\mathrm{p}<0.00001$ & - \\
CTR & $R=0.32$ & $R=0.32$ & $R=-0.22$ & $R=0.33$ \\
& $\mathrm{p}=0.002$ & $\mathrm{p}=0.002$ & $\mathrm{p}=0.03$ & $\mathrm{p}=0.001$ \\
\hline
\end{tabular}

CTR = cardiothoracic ratio; LVEDD = left ventricular end diastolic dimension; $\mathrm{LVEF}=$ left ventricular ejection fraction; LVESD $=$ left ventricular end systolic dimension.

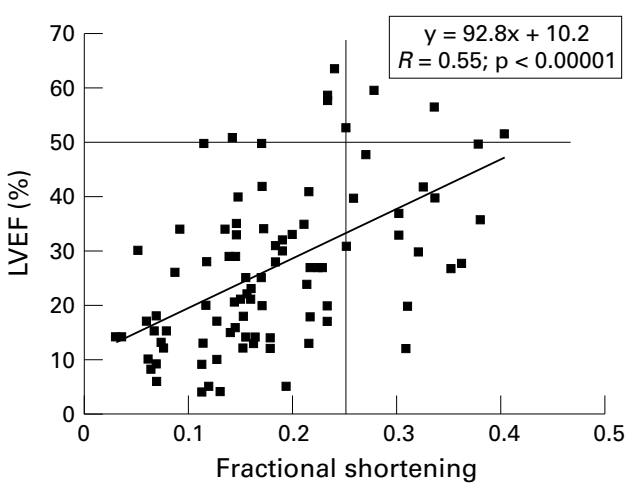

Figure 1 Relation between fractional shortening derived from echocardiography and left ventricular ejection fraction (LVEF) derived from radionuclide scanning. The lower end of the normal ranges are shown.

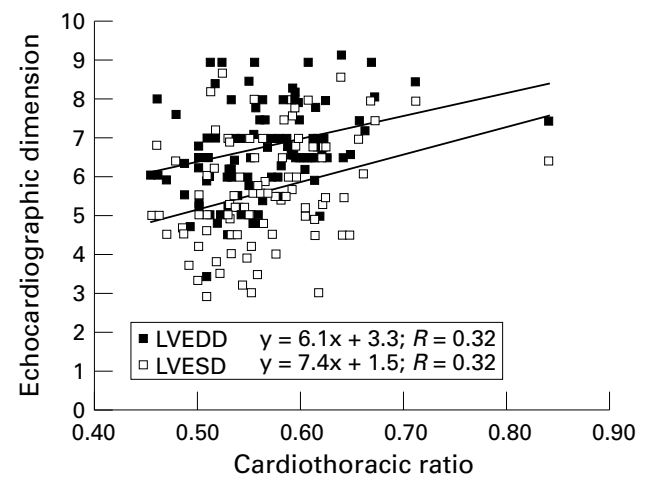

Figure 2 Relation between cardiothoracic ratio on a posteroanterior chest film and left ventricular end diastolic dimension (LVEDD) and left ventricular end systolic dimension (LVESD). 
There was no significant relation between exercise capacity (as assessed by peak oxygen consumption) and any of the indices of cardiac function (the strongest relation was between peak oxygen consumption and LVEF; $r=0.23$, $\mathrm{p}=0.05$ )

Twelve patients had a CTR in the normal range $(0.50)$. For this group, the LVEDD was slightly smaller than for the rest $(6.2(0.9) v 6.9$ (1.2); $\mathrm{p}=0.045)$, but the ejection fraction was significantly higher (35.6 (17.5) v 25.7 (14.2); $\mathrm{p}=0.03$ ).

\section{Discussion}

Establishing the diagnosis of chronic heart failure accurately is essential for the correct management of the patient. ${ }^{8}{ }^{9}$ Clinical examination alone is unreliable. ${ }^{12}$ The most widely available imaging method available is echocardiography, with the dimensions of the heart and fractional shortening being the most reliable methods for quantifying function. Ejection fraction can be estimated from long axis dimensions. These methods are limited by being unable to take account of regional abnormalities. ${ }^{10}$ More sophisticated methods are very dependent on image quality, are time consuming, and are not routinely available. Echocardiography can be limited by poor image quality, ${ }^{11}{ }^{12}$ although the impression of a trained operator is suitable clinically even in the absence of accurate measurements. ${ }^{13}$

Radionuclide scanning provides a measure with which clinicians are instinctively happy, LVEF. The correlation between echocardiography and radionuclide scanning has not been good in previous reports. ${ }^{10}{ }^{14} \mathrm{LVEF}$ from radionuclide ventriculography is more reproducible than echocardiography. ${ }^{15}{ }^{16}$ Radionuclide ventriculograms are less readily available than echocardiograms, and provide less overall information about the heart. Chest radiographs are commonly used as an initial test for the diagnosis of heart failure, particularly in general practice, ${ }^{17}$ but the apparent size of the heart on a plain film can be very misleading. ${ }^{18}$

The results reported here show that the correlations between heart size on radiography and left ventricular function measured by echocardiography and radionuclide ventriculography are too poor for the chest radiograph to be useful clinically as a diagnostic test of left ventricular function. Similar conclusions have been reported in studies postmyocardial infarction. ${ }^{19} 20$

Radionuclide scanning and echocardiography compared well with each other. There were a few patients with a low ejection fraction who were found to have normal fractional shortening, but these patients had enlarged left ventricles. There were six patients with a low fractional shortening who had a normal ejection fraction. The majority of patients were shown to have left ventricular impairment by both methods.

\section{CONCLUSIONS}

Chest radiography is an unsatisfactory method for diagnosing heart failure or drawing inferences as to cardiac function. The results of radionuclide ventriculography and echocardio- graphy in a routine clinical setting agree well with each other and are each appropriate diagnostic tools.

\section{LIMITATIONS}

This was a retrospective study. We have not included patients who have normal cardiac function. It is unethical to expose normal subjects to radionuclide scanning.

There is no absolute standard for the diagnosis of heart failure. The patients in this study were diagnosed on the pragmatic basis that they had symptoms compatible with heart failure in the presence of objective evidence of left ventricular dysfunction.

As this was a retrospective study, it is not possible to be certain of the standardisation for chest radiography. A poor inspiration can give a spuriously raised cardiothoracic ratio. The wide scatter of the points suggests that this has not been a systematic error.

1 Harlan WR, Oberman A, Grimm R, et al. Chronic congestive heart failure in coronary artery disease: clinical criteria. Arch Intern Med 1977;86:133-8.

2 Stevenson LW, Perloff JK. The limited reliability of physical signs for estimating hemodynamics in chronic heart failure. ҰAMA 1989;261:884-8.

3 McKee PA, Castelli WP, McNamara PM, et al. The natural history of congestive heart failure: the Framingham study. $N$ Engl F Med 1971;285:1441-6.

4 Carlson KJ, Lee DC, Goroll AH, et al. An analysis of physicians' reasons for prescribing long-term digitalis therapy in cians' reasons for prescribing long-term digi

5 Marantz PR, Tobin JN, Wassertheil-Smoller S, et al. The relationship between left ventricular systolic function and relationship between left ventricular systolic function and congestive heart failure

6 Pation 1988;77:607-12. prognosis in severe chronic heart failure. Am Heart f 1992; 123:421-6.

7 Cohn JN, Johnson GR, Shabetai R, et al. Ejection fraction, peak exercise oxygen consumption, cardiothoracic ratio, ventricular arrhythmias, and plasma norepinephrine as determinants of prognosis in heart failure. The V-HeFT VA Cooperative Studies Group. Circulation 1993;87(suppl):VI $5-16$.

8 Task Force on Heart Failure of the European Society of Cardiology. Guidelines for the diagnosis and assessment of heart failure. Eur Heart $\mathcal{F}$ 1995;16:741-51.

9 Task Force of the Working Group on Heart Failure of the European Society of Cardiology. The treatment of heart European Society of Cardiology. The
failure. Eur Heart $\mathcal{f}$ 1997;18:736-53.

10 Albin G, Rahko PS. Comparison of echocardiographic quantification of left ventricular ejection fraction to radionuclide angiography in patients with regional wall radionuclide angiography in patients with regional
motion abnormalities. Am 7 Cardiol 1990;65:1031-2.

11 Francis CM, Caruana L, Hearney P, et al. Open access echocardiography in management of heart failure in the community. BMF 1995;310:634-6.

12 Savage DD, Garrison RJ, Kannel WB, et al. Considerations in the use of echocardiography in epidemiology: the Framingham study. Hypertension 1987;9:40-4.

13 Choy A-MJ, Darbar D, Lang CC, et al. Detection of left ventricular dysfunction after acute myocardial infarction: comparison of clinical, echocardiographic, and neurohormonal methods. Br Heart f 1994;72:16-22.

14 Jensen-Urstad K, Bouvier F, Hojer J, et al. Comparison of different echocardiographic methods with radionuclide different echocardiographic methods with radionuclide imaging for measuring left ventricular ejection fraction during acute myocardial infarction treat
therapy. Am $\mathcal{f}$ Cardiol 1998;81:538-44. 15 Wackers FJ, Berger HJ, Johstone DE, et al. Multiple gated
cardiac blood pool imaging for left ventricular ejection cardiac blood pool imaging for left ventricular ejection fraction: validation of the technique and

16 Himelman RB, Cassidy MM, Landzberg JS, et al. Reproducibility of quantitative two-dimensional echocardiography. Am Heart f 1988;115:425-31.

17 Edep ME, Shah NB, Tateo I, et al. Difference in practice patterns in managing heart failure between cardiologists, family practitioners, and internists [abstract]. F $\mathrm{Am}$ Coll Cardiol 1996;27:367A.

18 Chakko CS, Woska D, Martinez H, et al. Clinical, radiographic, and hemodynamic correlations in chronic congestive heart failure. Conflicting results may lead to inappropriate care. Am $\mathcal{f}$ Med 1991;90:353-9.

19 Madsen EB, Gilpin E, Slutsky RA, et al. Usefulness of the chest x-ray for predicting abnormal left ventricular function after acute myocardial infarction. Am Heart $\mathcal{f} 1984 ; 108$ : 1431-6.

20 Alam M, Rosenhamer G, Höglund C. Comparability of Alam M, Rosenhamer G, Höglund C. Comparability of arction. F Intern Med 1989;226:171-5. 